\title{
Detection of genes involved in regulation of wheat flowering time
}

\author{
A.A. Kiseleva*, A.B. Shcherban, I.N. Leonova, E.A. Salina \\ Institute of Cytology and Genetics SB RAS, Novosibirsk, Russia \\ *e-mail: antkiseleva@bionet.nsc.ru
}

Key words: wheat, flowering time, SNP, putative transcription factors

Motivation and Aim: Variability of flowering time may assist in wheat adaptation to local environments. Thereafter, discovery of new flowering time determinants is important for cereal improvement. In this study, we used common wheat cultivar Chinese Spring (CS) and the substitution line of CS with 5B chromosome from T. dicoccoides (CS-5Bdic), different in their flowering time by two weeks, to detect determinants of flowering time on $5 \mathrm{~B}$ chromosome.

Methods and Algorithms: To ascertain the loci determining flowering time difference, a set of 116 recombinant inbred 5B chromosomal lines as a result of hybridization of CS with CS-5Bdic were developed and their flowering time was estimated. Genotyping was performed using the Illumina Infinium 15k Wheat platform and a set of SSR markers. Genetic linkage map was developed using the MultiPoint UltraDense software.

Using the QTL-analysis locus on 5B chromosome was revealed. To detect candidate genes functional annotation of genes, associated with SNP markers, based on synteny with other plant species was performed.

Results: Phenotype (flowering time) - genotype association analysis revealed 79 markers in pericentromeric region of $5 \mathrm{~B}$ chromosome significantly associated with flowering time variation. Based on SNP sequences and synteny with crop genomes we identified the four best candidate genes: WRKY, ERF/AP2, FHY3/FAR1 and ELF4, known to be involved in flowering time modulation in model species.

Conclusion: We identified the four best candidate genes: WRKY, ERF/AP2, FHY3/ FAR1 and ELF4. These genes were previously shown to be involved in flowering time modulation. We propose that the probable cause of flowering time differences may be due to differences in the origin of interacting flowering time pathways and putative transcription factors located on 5B might modulate. Contribution of FHY3/FAR1 in flowering pathways was shown in further experiments.

Acknowledgements: This study was supported by the RSF (Project No. 14-14-00161). 